VP-13

\title{
Total robotic pancreatoduodenectomy with pancreatico-jejunostomy by modified Blumgart technique
}

\author{
Vaibhav Kumar VARSHNEY*, Sunita SUMAN
}

Surgical Gastroenterology, All India Institute of Medical Sciences, Jodhpur, Rajasthan, India

Introduction: Robotic pancreatoduodenectomy (RPD) has gained ground in recent past. Pancreaticojejunostomy with modified Blumgart technique is less often used with minimally invasive approach due to its technical complexity, however it is feasible with robotics due to its multi-arm platform, three-dimensional view and an extended range of motion. We describe our approach for total robotic pancreatoduodenectomy with pancreatojejunostomy by modified Blumgart technique in this video.

Methods: Patient placed in reverse trendelenburg position with leg-split posture. Two port on each side of umbilicus $8 \mathrm{~mm}$ apart in mid-clavicular line and anterior axillary line with infra-umbilical port $(12 \mathrm{~mm})$ work as an assistant port. The dissection phase is performed in a standard manner with extended kocherisation, early division of antrum, caudal-cranial dissection of uncinate process. Pancreatico-jejunostomy: Transpancreatic horizontal mattress sutures are placed to secure the pancreatic parenchyma to the jejunum using 3-0 prolene. After a small enterotomy, a series of interrupted sutures with 5-0 PDS are used for duct to mucosa anastomosis. Finally, anterior layer of 3-0 prolene buttress sutures completes the anastomosis. Hepaticojejunostomy by Kelly's technique and gastrojejunostomy either stapled or hand sewn after extraction of specimen. Feeding jejunostomy was performed.

Results: Patient did well in the post-operative period and doing well after 1 year of follow up.

Conclusions: Robotic pancreatoduodenectomy with pancreatojejunostomy using modified Blumgart technique is feasible with robotic arms. 